\title{
Open Innovation in Response to Covid 19 :A case study of the Open Source Ventilator Ireland Project
}

\author{
Colin Keogh ${ }^{1}$ \\ 1 University College Dublin
}

Funding: The author(s) received no specific funding for this work.

Potential competing interests: The author(s) declared that no potential competing interests exist.

\section{Abstract}

The sudden onset of Coronavirus (SARS-Cov-2) resulted in the creation of large scale informal innovation networks to tackle a lack of critical medical equipment resulting from overwhelmed healthcare systems. Recent developments in distributed manufacturing, remote collaboration and the popularisation of open innovation concepts, added to a human desire to help tackle this pandemic, allowed the generation of innovations at scales, paces and impacts never seen before. This research looks at one such informal innovation network, [1], discussing its formation, development and impacts achieved. A review of the external moderators of the project is conducted, assessing the significant external influences impact its development, followed by an internal management review of the project from an operational viewpoint. The final aspect is a mapping exercise, influences by Chesbrough's Open Innovation Model, which results in a proposed new version of this model, "The Inverted Open Innovation Distribution Model" derived from analysis of the TeamOSV process in response to Coronavirus (SARS-Cov-2). Future work looking at approach specific policies, regulations, mechanisms and pathways is needed to help increase the potential impact of not open source ventilator projects, but other emergency response projects in the future.

\section{Introduction}

Innovation thrives during crisis, be it a World War, a Trade War or a war against a novel virial pathogen leading to a global pandemic. Coronavirus (SARS-Cov-2) has grown rapidly around the world, with as of 30 August 2020, infected some 25 million people, resulting in an estimated ${ }^{2}$ ]. This is the worst global pandemic since the Spanish flu, reaching Europe in late February/Early March 2020, spreading rapidly throughout populations.

During the earliest stages of the pandemic (Beginning around March 2020), the world was faced with the prospect of overwhelmed medical infrastructure, at regional and national levels, due to the highly infectious nature of SARS-Cov$2^{[3],[4],[5]}$. Overwhelmed medical infrastructure due to the influx of SARS-Cov-2 patients into regional healthcare systems, resulted in spiking mortality rates in patients, notable in the Lombardy region of Italy ${ }^{[6]}$. Traditional medical infrastructure possesses adequate supply of critical medical equipment, but not in high enough density to handle a significantly increased volume of patients resulting from pandemics ${ }^{[7]}$. This lack of critical care equipment results in unnecessary deaths and infections of both patients and hospital staff. Ventilators, Personal Protective Equipment (PPE) and Patient 
Communication Systems are some examples of the types of technologies that were in short supply during the early stages of the SARS-Cov-2 pandemic.

In response to the rapidly increasing infections of coronavirus, a global response from Scientists, Engineers, Makers and other non-medical professionals was born, with the goal of utilising Open-Source technology and a naturally arising form of Open Innovation to help support humanities response to SARS-COV-2. Thankfully, the recent development and deployment of open source small scale manufacturing technologies, increasing growth of free open-source technical software, and growing popularity of remote collaboration platforms (also increased significantly due to pandemic related lockdowns) have allowed the forming of informal innovation networks to tackle large scale complex challenges. In March 2020 , many of these informal networks were forms in response to SARS-COV-2, with this work assessing the development, impact and implications of one such network.

\section{Open Source Ventilator Ireland}

One of the many Open-Source responses to this perceived lack of critical medical equipment was the Open Source Ventilator Ireland group establish in Mid-March 2020. Open Source Ventilator (OSV) ${ }^{[8]}$ Ireland was formed initially with the goal of building a focus team in Ireland to begin development on what was termed the "Field Emergency Ventilator (FEV)". Inspired by the initial efforts of the Open Source COVID-19 Medical Supplies (OSCMS), which initially focused on developing open ventilators but quickly refocusing mainly on the local production of Personal Protective Experiment (PPE). OSV Ireland partnered with the OpenLung team ${ }^{[9]}$ in Canada, who were developing and publishing open source designs via GitLab. The group quickly grew via online engagement and media attention, amassing volunteer engineers, designers and medical professionals with the goal of developing new, low resource medical interventions to support a perceives lack of mechanical ventilation equipment globally. The well-known Bag Valve Mask (BVM) quickly became the core functional component of the OSV design, with the goal of utilising 3D printed and traditionally manufactured components for localised assembly of the proposed systems to maximise potential manufacturing capabilities around the globe. The inherent ability of 3D printing to easily share design files, and rapidly product prototypes were just two to the significant benefits it offered to this use case.

The group grew rapidly, onboarding thousands of members to their open slack group, to facilitate the rapid open-source innovation of new mechanical ventilator concepts. It quickly became apparent that there also existed shortages in other critical medical equipment, such as Personal Protective Equipment (PPE) and educational material aimed at the general public regarding SARS-COV-2. An additional slack channel was started, Open Source Ventilator Extended (OSVX) to focus on the innovation and development of other non-ventilators. This additional group focus on the collection, structuring and identification of needs and requirements from frontline staff, allowing the development of additional supports. Both arms of the project (OSV \& OSVX) were realigned under the unified TeamOSV structure, with new branding, structures and websites developed. This expansion, adaption and unification arouse from the ever increasing needs and shortages experiences by the medical field due to SARS-COV-2. At its peak, TeamOSV gathered over 3,000 volunteers into an open-source multidisciplinary team of engineers, designers, makers, and medical practitioners working on developing not 
only new ventilator concepts but also 30 other novel low-resource interventions through online collaboration to support the fight against SARS-COV-2.

\section{Evolution of an Ad-hoc Open Innovation Network}

The TeamOSV group as established by its co-founders, was heavily influenced by their professional experiences with the Innovation, Academic Research, Humanitarian, Technology and Start-up fields [10][11][12][13][14], thus was heavily influenced by the application and impact of 3D Printing technology and its impact on the traditional manufacturing process. A new form of manufacturing paradigm known as "Mass Distributed Manufacturing", arose from the recent developments in open-source small scale manufacturing systems ${ }^{[15]}$. As discussed by Pearce ${ }^{[16]}$, in this new form of production, designs are developed and shared under open source licenses online, thus allowing others to download and replicate these designs using their own equipment, even in domestic households ${ }^{[17]}$. This new method has resulted in ongoing successful hardware production and distribution, particularly in when lower cost customised equipment is required, including the production of open-source scientific hardware for use in lab environments ${ }^{[18]}$, E-Nable low cost $3 d$ printed prosthetic hands ${ }^{[19]}$ and 3D printing of artefacts for Marine protection activities ${ }^{[13]}$. These successes, in particular in the application for scientific hardware, showed significant opportunity to apply the open source design principles ${ }^{[20]}$ along with mass collaborative distributed manufacturing to produce necessary medical equipment in response to SARS-COV-2. Inspired and supported by this previous work, Open Source Ventilator Ireland (and subsequently TeamOSV) focused it efforts on the development of open source medical interventions, solutions and innovations to help overcome SARS-COV-2 resulted ventilator, equipment and medical supply shortages ${ }^{[21]}$.

\section{Operational Dynamics of TeamOSV}

From its inception, the TeamOSV network operated fully remotely, due to the geographical spread of its contributors, and rapidly growing movement restrictions placed on society due to SARS-COV-2. It was initially inspired by similar efforts beginning on Facebook, namely the Open Source Medical Supplies Group (OSMS) ${ }^{[22]}$ started in early March 2020, leading to a more centralised effort focused on ventilator production led from Dublin, Ireland. The group grew rapidly, initially opening an expression of interest form on the original OSV website ${ }^{[8]}$, which resulted in over 6,000 unique expressions of interest (up to July 2020), with rapid growth seen during the initial stages pandemic growth in Europe and the United States, as seen in Figure $1^{[1]}$. These EOl's came from 144 Countries, spanning 6 continents, with nearly 5,500 submission before the 30 March 2020. This website based EOI approach was taken in order to funnel volunteers into the projects newly established Slack channel. 


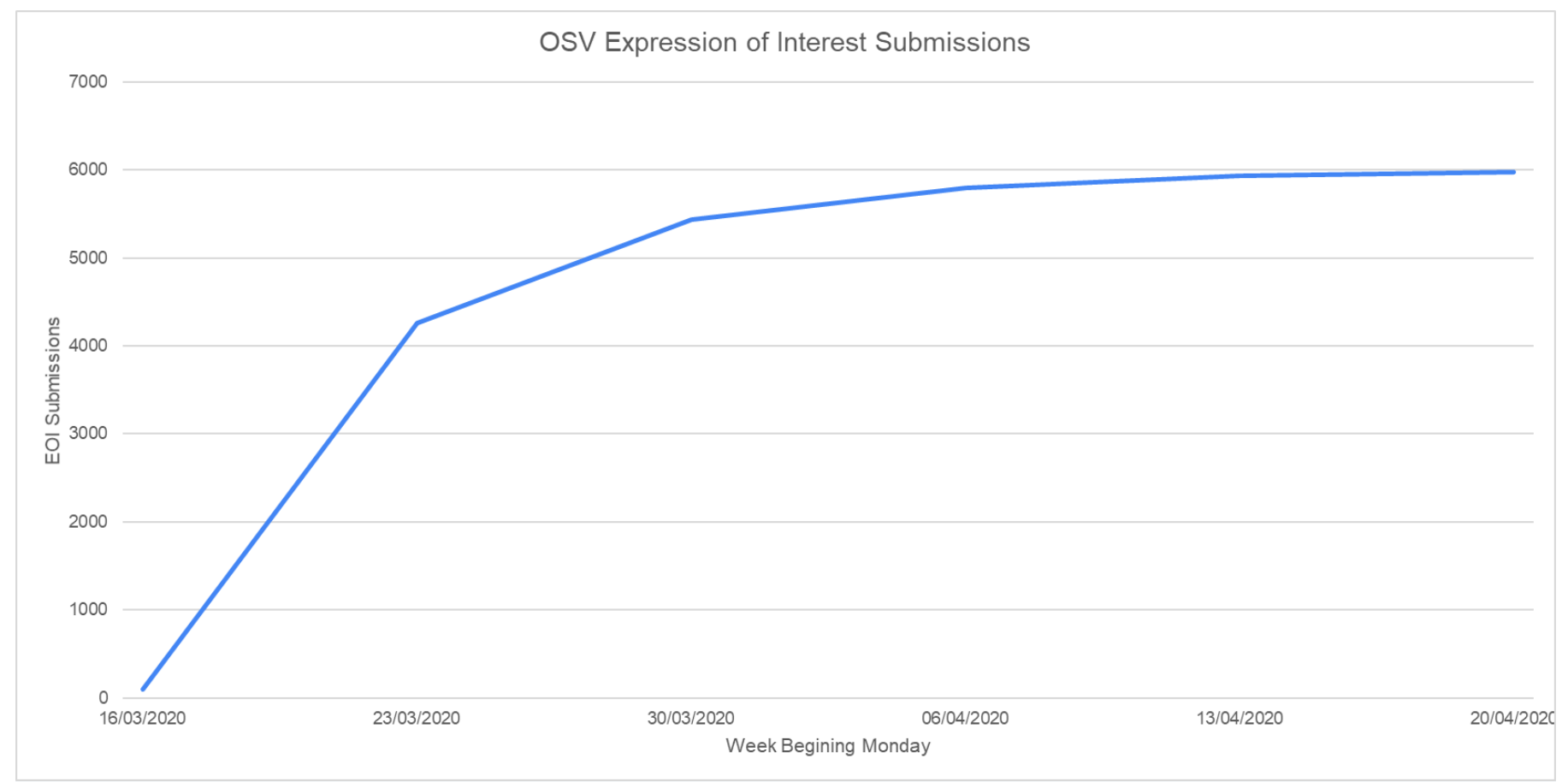

Figure 1. Open Source Ventilator Expressions of Interest (Week Periods from 16 March - 20 April) ${ }^{[1]}$

The main functional element of TeamOSV was its Slack channels, which were established to centralise efforts, enhance information sharing and aid in remote collaborative development. The first channel focused on the development of the ventilator related projects which was open on the $15^{\text {th }}$ March 2020[23], with the second channel focused on all nonventilator projects which was opened on the $21^{\text {st }}$ of March $2020^{[24]}$. The OSV channel resulted 36 public channels and a total of 2,508 members as of 20 August 2020. It generated 54,595 messages, 9 GB of data with a peak of 785 active daily members (23 ${ }^{\text {rd }}$ March 2020). The OSV-X channel resulted 42 public channels and a total of 1,418 members as of 20 August 2020. It generated 44,415 messages, 4 GB of data with a peak of 235 active daily members ( $7^{\text {th }}$ April 2020). These slack groups were fully open, accessible and available, with all designs, data and information shared freely between members. All developed solutions and innovation were shared regularly via the project website, social media channels, internal slack channels and other file repositories. Social media had played a significant role in the distribution information (and disinformation), so integration of social media promotion was a key part in disseminating the outcome of the project, finding project contributors and connecting with other relevant projects.

The ventilator arm of this project also utilised the OpenLung $\mathrm{Gitlab}^{[9]}$, as seen in Figure $2^{[9]}$, a web based DevOps lifecycle tools, acting as a git repository manger to allow all contributors to access operational instructions, updates, the development pipeline, relevant design files while also providing real time issue tracking. While Git repositories are traditionally used for software development to track changes and versions of code bases, It was utilised in this case as a fully public access file repository and collaborative hardware development tool, all under the GNU General Public opensource licence. This licence is a free copyleft license for software and other kinds of works. As TeamOSV and OpenLung are sharing digital design files, not physical objects, this was chosen as the most appropriate license. 


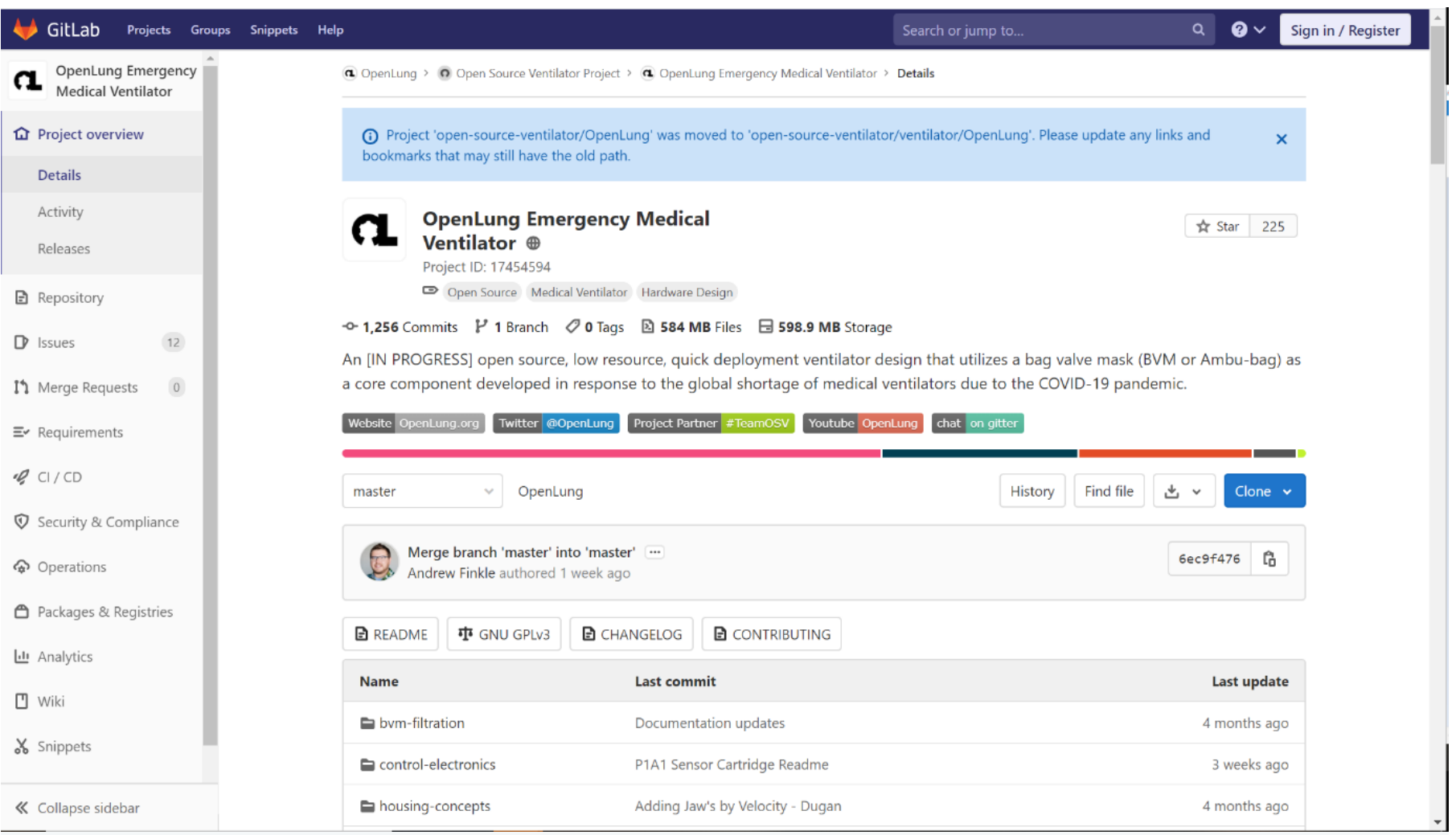

Figure A. GitLab Repository Utilized as an Open-Source Hardware Development Tool ${ }^{[9]}$

Due to the diverse range of skillsets, the global distribution of teams and the relatively limited interaction between teams, TeamOSV \& OpenLung set out a basic open-source and open innovation inspired project philosophy. This philosophy was based on the project being (or aimed at the):

- Open source in nature so iterative and open for community involvement

- Development of a Field Emergency Ventilator (FEV) to support medical practitioners

- Development of other Frontline care requirements such as PPE, Applications and other medical tools.

- Safety is paramount. These concepts are designed to reduce harm and risk of death. No potentially unsafe devices will be shared. Medical validation is a priority.

- Supplies and materials will be locally sourced where available

- The design(s) will be validated; validation will be documented, and transparency will be made available to local health service.

- Dissemination via Internet (www.TeamOSV.com) and GitLab.

A number of basic operating assumptions were set to also support the project development, namely; that Regulatory bodies may waive clearance or reduce regulatory barriers for emergency interventions", "Traditional certified medical components and supplies used in ventilators will be in short supply" and "Logistics will be impaired/disrupted. Non-medical supplies, manufacturing systems and components will be readily available globally"[8]. The guiding philosophy and the list 
of initial operating assumptions set the initial goals of the project, setting some boundary conditions for the work for all volunteers. These conditions took the place of a standardised set of guideline specifications, which the first of which were release on $20^{\text {th }}$ of March 2020, thus allowing development to start in earnest. These guidelines were a core requirement for effective open source ventilator generation, but also a major hurdle in the progression of may open source projects, which is discussed in detail in subsequent sections.

\section{$\underline{\text { Outcomes }}$}

TeamOSV core activities ran from March 2020 until July 2020, with progress slowing as the ventilator shortage never materialised and the provision of other medical equipment tackled localised shortages. Over the space of approximately 5 months, TeamOSV volunteers made significant impacts in Ireland and around the world, in response to SARS-COV-2. The ventilator arm OSV, produced a total of 21 conceptual ventilator designs, example of which can be seen in Figure $3^{[8]}$, 5 of which made it to final design with another 2 progressing towards testing. Testing occurred in the United States, producing significant operational insights, parameters and necessary requirements.

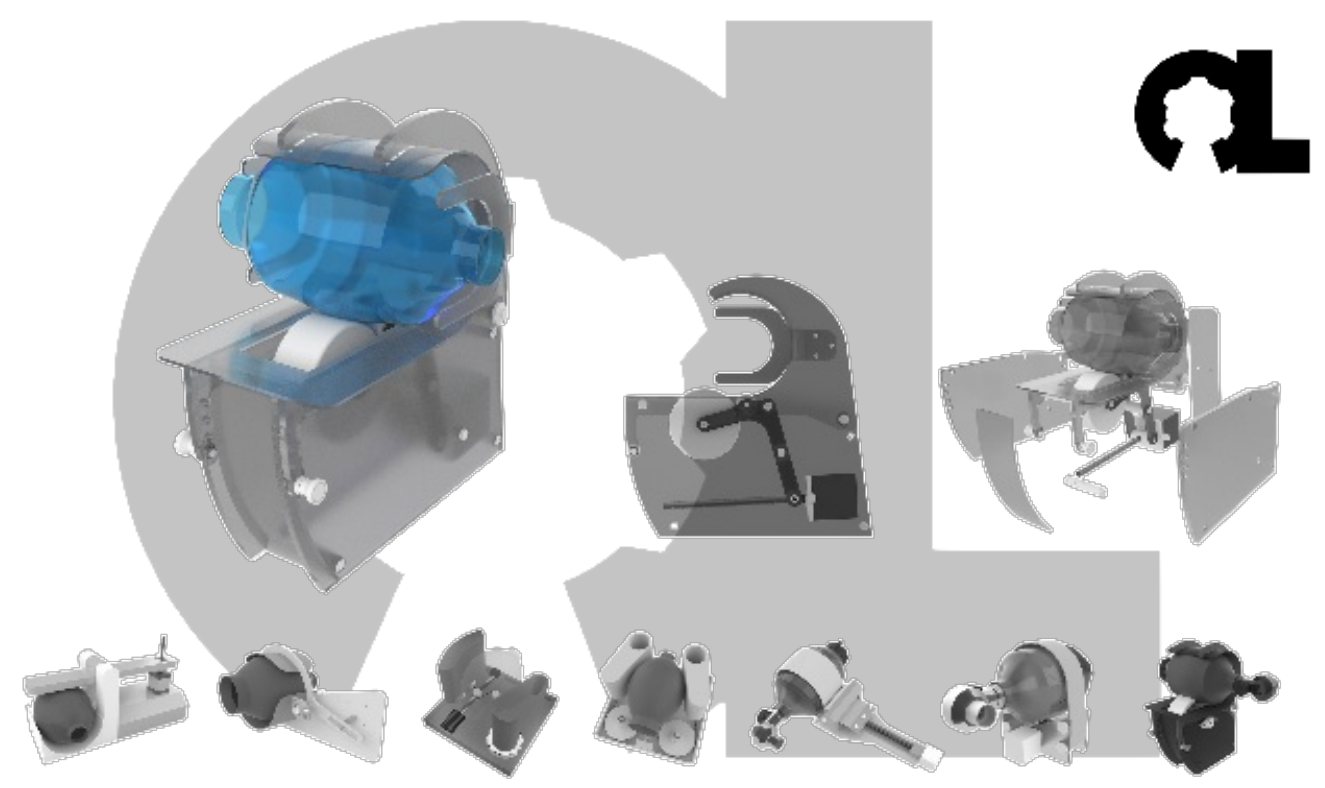



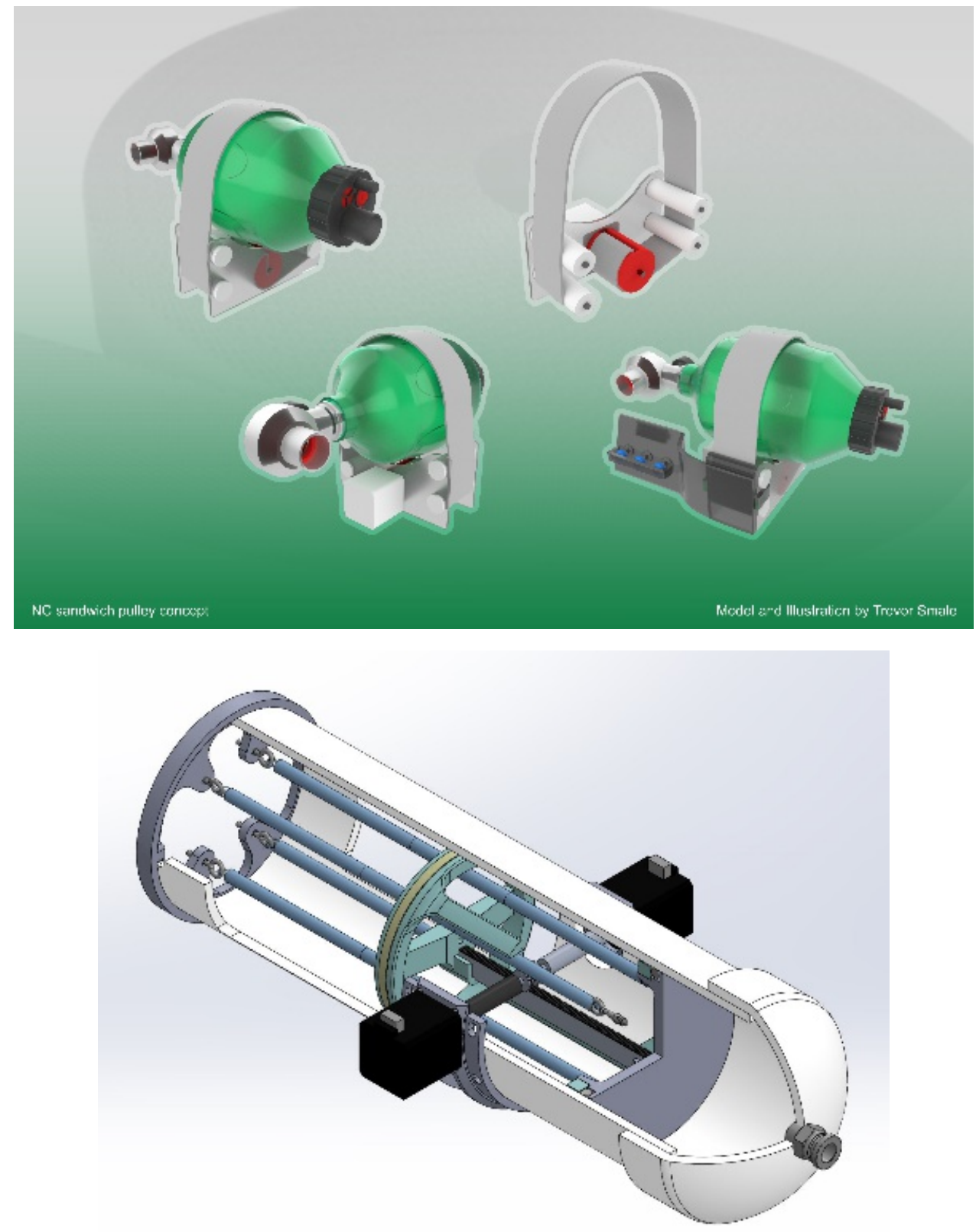

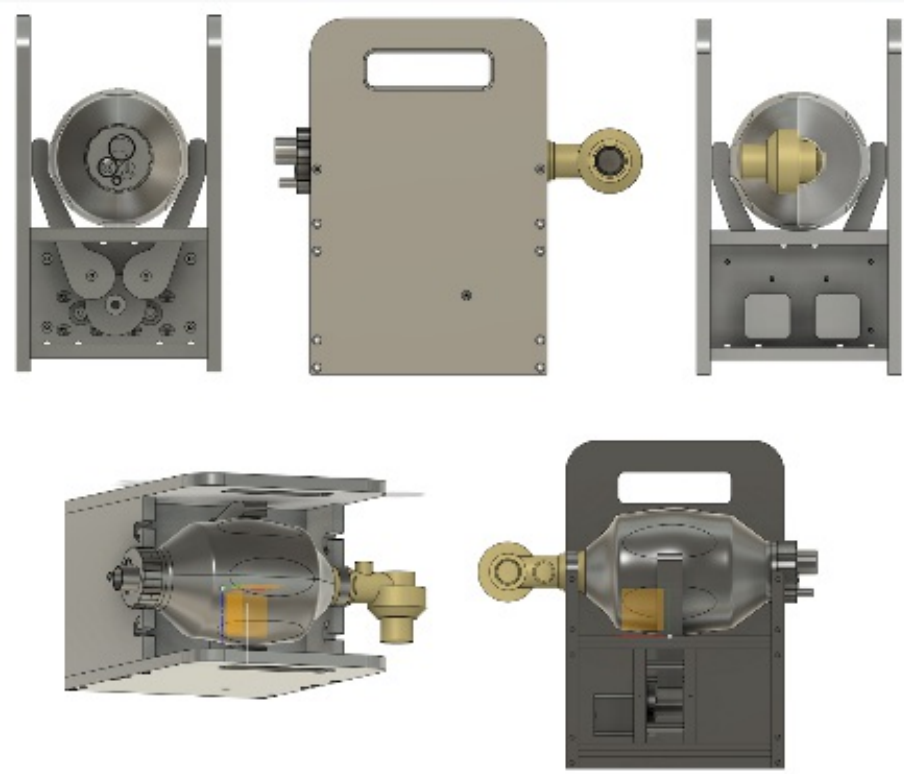

Figure 3. Examples of Ventilator Concepts Developed under the TeamOSV Banner ${ }^{[8]}$

In addition to ventilator design concepts, a number of ancillary systems were also developed including Control Electronics, Sensor Layouts \& Specifications, Housing Concepts, Humidifier Concepts \& Filtration considerations. Regulatory guidance was developed and catalogued, Documentation provided and changelogs accessible for revision control. All of these details can be found on the OpenLung GitLab ${ }^{[8]}$. These concepts inspired and support localized development groups around the world including DIY Beatmungsgeraet in Germany ${ }^{[25]}$, Inspire OpenLung in Brazi[ ${ }^{[26]}$ and the Velocity Research team in the Unites States ${ }^{[27]}$.

Due to changing treatment protocols ${ }^{[28]}$, increasing production of critical care ventilators under the US Defense Production Act $^{[29]}$ and less severe surges of SARS-COV-2 than predicted, the predicted global ventilator shortage never materialised. As such TeamOSV development of open source ventilators has ceased. All files and design were made available on their websites, GitLab and Slack channel. The files are made available to help support other future projects, and to act as a repository if additional sever waves of SARS-COV-2 result in a resurgence of need for these ventilators.

The non-ventilator arm of TeamOSV, OSV-X achieved similar successes with a number of projects being developed to near or total completion. Thousands of face shields were produced and distributed free of charge to hospitals, care homes and other medical settings around Ireland, supported by detailed guidance on manufacturing $\&$ distribution ${ }^{[1]}$. Production of face mask straps, and other equipment to support PPE use was successfully delivered. Research and guidance documents were published in relation to face mask usage, efficacy and impact, along with manufacturing plans for the production of low cost disposable googles. Accessible easy to use information, guidance \& educational content was developed to help educate both medical professionals and the public on issues related to SARS-COV-2, examples of which can be seen in Figure $4^{[30]}$. Any problems which were not completed over the period of operation where turned into challenge information posters, for use by future projects, programs or organisations ${ }^{[1]}$. These innovations have found 
significant use globally, supporting the response and adaption to SARS-COV-2.

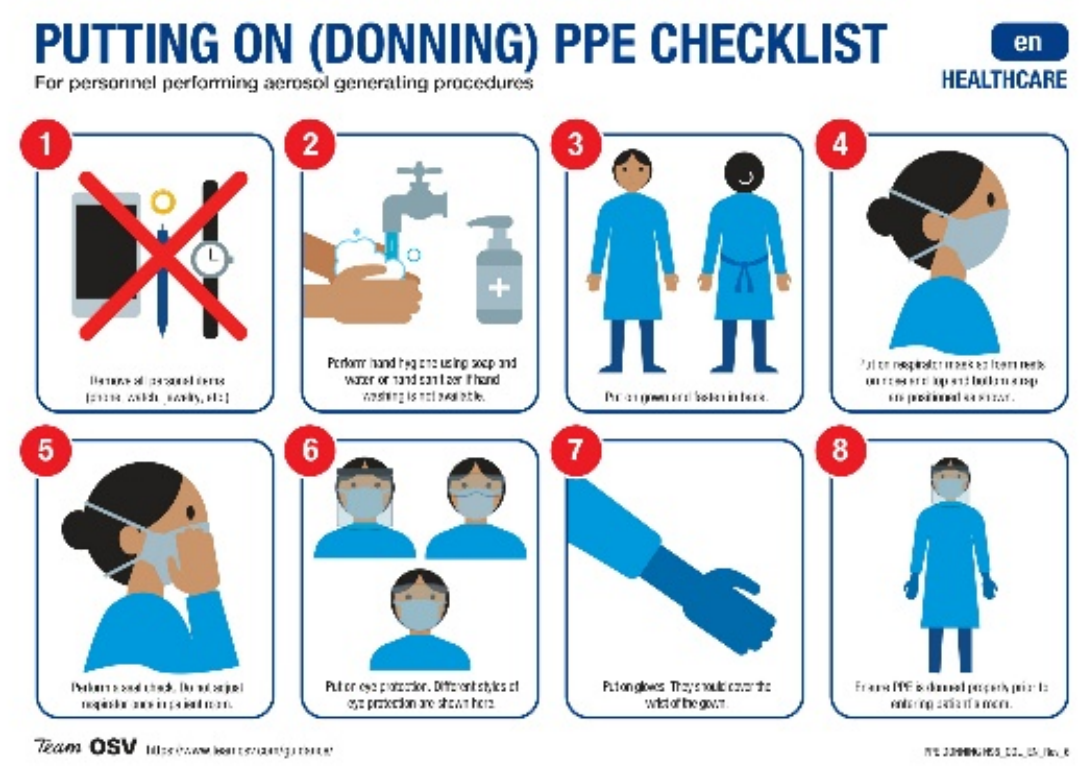

COMING HOME CHECKLIST
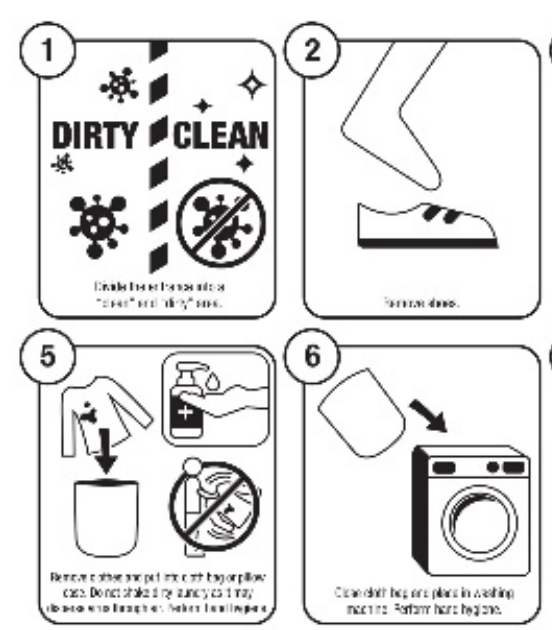

Team OSV

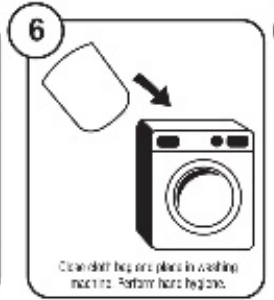

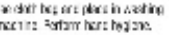
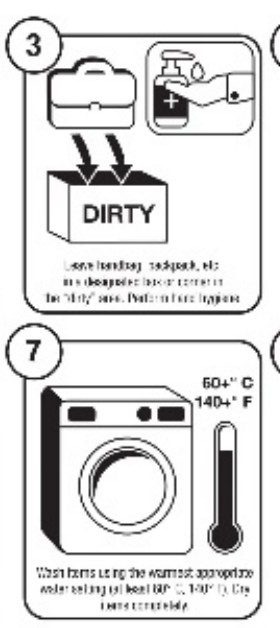

instemelele
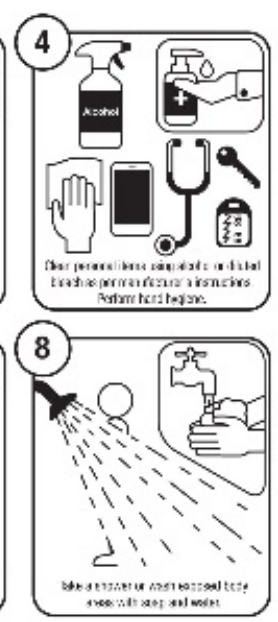

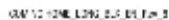

Figure 4. Exmaples of Produced Educational Material ${ }^{[30]}$

The final aspect of TeamOSV operations, was its project partner, Bravo Charlie Tango (BCT $)^{[31]}$, a group of volunteer Irish motorcycle riders worked to deliver emergency medical supplies to Hospitals/Medical Centres/Care Homes around Ireland to assist in fighting SARS-COV-2. BCT collaborated with another ad-hoc response group, Covid Community Response to allow the public to donate surplus PPE to Irish healthcare institutions through an online matching and motorbike delivery service. This service allowed the BCT team to safely collect and deliver hundreds of donations of PPE, 3D printing materials, manufactured face shields and other items to locations all over the Island of Ireland. 


\section{Crisis Driven External Project Moderators}

As in any crisis, the core aspects change and evolve at lightning pace. This was true for the open source response to SARS-COV-2. The ever changing inputs, pressures and influencing factors generated a number of significant external project moderators at every stage of the SARS-COV-2 pandemic. A number of the most impactful external project moderator are discussed below. In the early stages of this open source response, rolling lockdowns and closures of Offices, Universities and Business significantly impacted the pace of open source developments.

As the group was established and operated from Dublin, Ireland, the impact of SARS-COV-2 restrictions effected the group as early as $12^{\text {th }}$ March $2020^{[32]}$. On this date the Irish government closed all schools and colleges due to the SARS-COV-2 public health emergency, quickly flowed by the closure of bars and public houses on the $15^{\text {th }}$ March $2020^{[33]}$. On the $27^{\text {th }}$ March, Irelands Taoiseach (Prime Minister) announced a series of lockdowns for Ireland, limiting movement, business operations and other societal activities, which are (as of 30 August 2020) still in effect in some form ${ }^{[33]}$.These lockdowns, movement restrictions and changes to business operations drastically slowed the development of the OSV responses. Many of the core engineering team in Ireland had their offices, workshops and labs closed due to the public health emergency, deliveries and availability of key components were significantly delayed and travel restrictions limited movement to testing and validations resource. To combat these issues, OSV activities moved in person production and development from locked down regions to open global regions as restrictions took hold. When Europe began to lockdown, development moved west to North \& South America, with logistics and remote development still underway in Europe. The pace of innovation would have been faster if impacts were avoided. While development moved global, many groups operating in this space looked towards local medical regulation bodies for guidance of non-standard ventilator production.

The concept of developing and deploying a low cost rapidly development medical device was unthinkable prior to SARSCOV-2. Medical device design is a highly regulation, complex and long term process to ensure the utmost levels of safety and efficacy. As such when it appeared that the world would face significant ventilators shortages ${ }^{[34]}$, specifications or standards for a rapidly developed low resource or open source ventilator did not exist. The British Government launched on the $15^{\text {th }}$ March $2020^{[35]}$ an initiative to support non-medical manufacturers in the United Kingdom to produce ventilators in the fight against SARS-COV-2. Work on setting out required specifications for manufacturers began, with input from numerous clinicians and medical professional, culminating in the release of the first formal specifications by the Medicines and Healthcare products Regulatory Agency (MHRA) on $20^{\text {th }}$ March 2020[36] . These specifications set out the "minimally acceptable" performance of such a device, with the intended purpose of the device; "short-term stabilisation for a few hours, extendable for up to 24 hours in extremis"[37]. This guidance formed the basis of the majority of the worlds open-source or rapidly designed ventilators, with many teams tackling this challenge ${ }^{[38],[39]}$. The Bluesky consortium was one such team, which partnered with Red Bull and Renault formula one racing teams to develop new ventilator systems $^{[40]}$.

Unexpectedly on $10^{\text {th }}$ April 2020, the MHRA updated it guidance to manufacturers, significantly increasing the required specifications of such devices, noting that "the greater proportion of devices.....must be capable of supporting 
spontaneous breathing modes" with additional requirements in smart sensing and fluid removal. These changes drastic raised the bar for approval in Europe, resulting in significant challenge to the establish ventilator projects, including Bluesky which ended its operation on the $11^{\text {th }}$ April 2020 with the UK government cancelling the orders it placed with Bluesky for ventilator systems ${ }^{[41]}$. While the governmental position is that "Clinical need" changed, resulting in the increased requirements, when in reality the initial specifications were too low for functional usage resulting from the initial rush to secure adequate equipment. These specifications acted as initial guidance for teams to target their devices desired performance, with all teams working in the wrong direction due to the inaccuracy and unsuitableness of this guidance.

An additional external moderator for this work was the significant media \& public interest in ventilators and particular in open-source based version. During the months of March and April, significant global interest was focused on ventilator production and procurement. The expected huge surges of SARS-COV-2 forced governments and healthcare providers to procure adequate supplies of ventilators and whip up a media storm regarding ventilators. Numerous media articles ${ }^{[34]}$ predicted shortages, with a large number of ventilator teams racing towards FDA Exception Use Authorization (EUA). This in effect produced a "Ventilator Arms Race" with teams racing to be the first with a certified design. As of the $14^{\text {th }}$ August 2020, the "Analysis of Open Source COVID-19 Pandemic Ventilator Projects ${ }^{[42]}$ shows 137 different open source pandemic ventilators, in various states of completeness. As of the $25^{\text {th }}$ March 2020, this list showed 24 different projects, as seen in Figure $5^{[42]}$. The external media and public pressure slowed the overall development of these systems, with teams holding back testing and development information from other groups. While most teams were fully open source, others closed source the development again slowing the overall development. The first "emergency" ventilator to obtain an EUA certification was the CoLabs COVIS Ventor system on the $4^{\text {th }}$ June 2020 , with many other certified since. While highly unlikely, it is currently unknown if any of these systems have every been used to treat a patient with SARS-COV-2. 


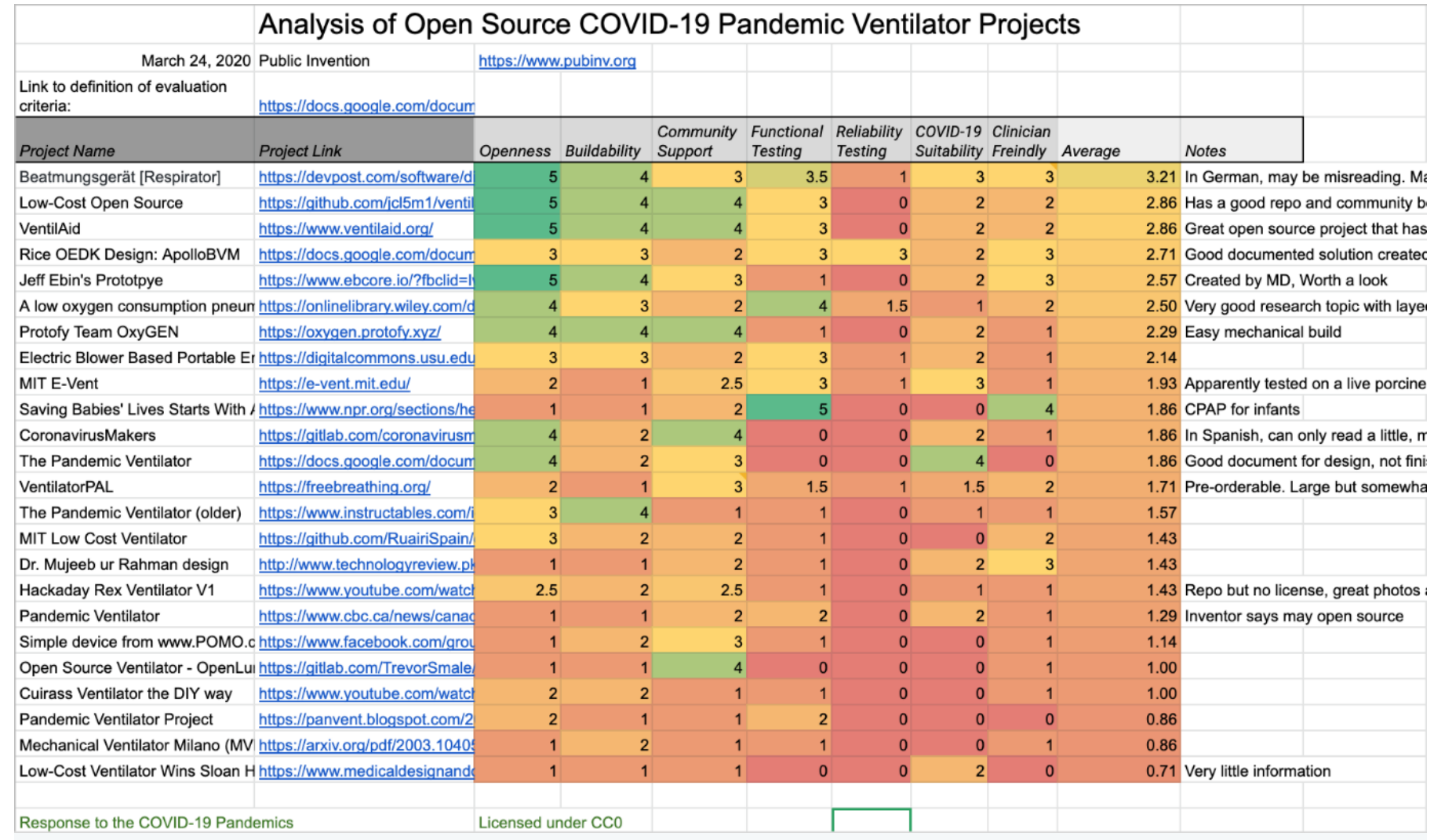

Figure 5. Analysis of Open Source COVID-19 Pandemic Ventilator Projects as of $25^{\text {th }}$ March 2020[42]

These external factors were unknown to everyone embarking on the challenge of developing these systems, in many cases significantly slowing or completing halting progress until solutions were found. In many cases reducing need, lack of potential use and the increasing need for complexity resulting in many projects being halted.

\section{Applied Innovation Management during a Crisis}

As the TeamOSV project evolved, a number of interesting innovation management lessons arose during the course of the project. The initial early stages of the TeamOSV network were chaotic and fractured, with order and structure only developing slowing over the course of a number of weeks. The pace of hardware development, which was amplified by the significant external need for these innovations resulted in a lack established organisational structures early in the project. Many non-expert or inexperience contributors suffered from a lack of focus and direction, with many possessing a desire to help but with no experience to know how they could help. This lack of support may have resulted in them disengaging from the group. This lack of initial structure resulted from the suddenness and apparent scale of the problem, the rapid growth in volunteers, a rapid increase in the volume \& variety of innovations considered and a lack of familiarity of the open source process by many volunteers. The voluntary nature of the work, added to difficulties in evaluating individual contributors' skills and experiences, along with the importance of safety \& regulations in the innovation being development resulted in difficulties in asserting an overarching hierarchy on both a macro and micro scale. While an overarching hierarchy (Core Team, Project Managers, Projects Leads etc.) was eventually established, the time taken to 
develop this structure resulted in confusion, repetition of work and this impacted the eventual outcome. Future projects of this type must develop a robust, clearly defined organisational structure and convey this structure to all relevant parties as soon as possible to avoid such conflict.

Conflict, namely conflict resolutions was also a vital component of this work. Many different aspects added to the potential for conflict, heightened emotions due to the external stressors of the pandemic, illness and lockdowns; a lack of experience in design/development projects, in open-source projects or in innovation work in general; a lack of face to face interaction and general personality classes and misplaced egos all resulted in raised tensions. Confliction resolution is a key part of such as project, with an understanding of the persona situations of the people involved required. Future projects should establish clear conflict resolution processes, circulate them to all involved and highlight the importance of interpersonal communication in all parties of the project.

The issue of project focus also arose during this work. The initial project focus was the development of Open Source Ventilators, with all efforts directed to that goal. The addition of non-ventilator projects, while very much needed, caused unnecessary confusion, conflict and separation between both project elements. Early in the process of onboarding new project ideas, a decision must be made whether to integrate or separate the different projects, therefore not compromising both for the sake of the other. Similarly, personal focus and volunteer motivations must be made clear early on to all involved.

While Intellectual property was not a major concern during the course of this work, the use and application of derived solutions going forward is a potential concern. As per Chesbrough ${ }^{[43]}$, there is a need for participant to consider the impact of hoarding their own developed IP or holding back innovations for future personal utilisation. Whether through malice or a lack of experience, a reluctance to share the innovations and ideas developed in an open and timely fashion impeded the work undertaken. In the future, there needs to be external mechanisms and platforms in place for the open collection, distribution and sharing of these open innovation outcomes, thus protecting the outcomes from internally or externally derived harm. As significant support would be an external agency, such as the WHO, who would act as an open repository for information, for the benefit of all organizations globally, as per Chesbourgh ${ }^{[43]}$.

\section{Mapping the TeamOSV Innovation Journey}

In order to better understand the process undertaken by the TeamOSV project, the mapping of this open innovation project was conducted. Utilizing the Open Innovation Model[ ${ }^{[43]}$, which defines open innovation as a model in which firms commercialise external ideas by deploying outside (along with inside) pathways to the market ${ }^{[44]}$, with the use of purposefully inflows and outflows of knowledge to accelerate internal innovation and expand the markets for external use of innovation ${ }^{[45]}$. While this model focuses on the development of new ideas for business gain in a particular market, the operational dynamics of this model are highly relevant to this response to SARS-COV-2. Utilising the Open Innovation Model[ ${ }^{[45]}$, as seen in Figure 6, as a functional model base, the individual elements of where mapped to the challenge of trying to develop and open source ventilator remotely with an assembled group of volunteers. 


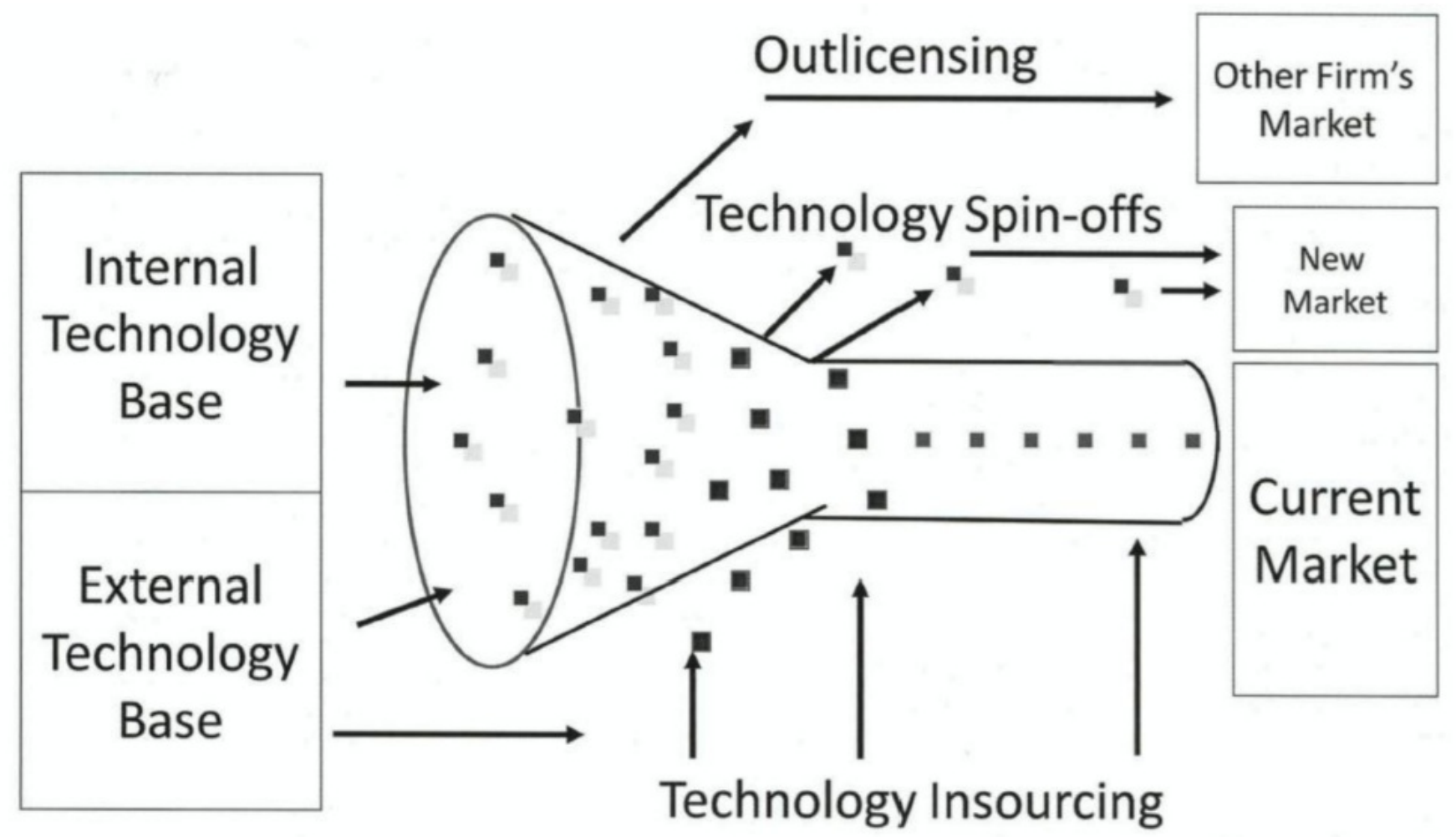

$\mathrm{R}$

D

Figure 6. The Open Innovation Model[ ${ }^{[46]}$

While the Open Innovation model takes wide ranging influence from both internal and external technology bases, feeding ideas and innovation into the funnel during the research phase, in this situation a concrete need was clearly defined and validation by external drivers prior to project inception (i.e.. Production of a low cost open source ventilator). The clearly defined goal is then assessed and analysed by the innovator (s) considering the external project influences (Issue Moderators), currently available solutions (External IP) and technology available to the problem in question (Technology Insourcing). The problem and the field it inhabits then reaches a boundary condition, in the case of TeamOSV that was current medical device \& equipment regulations, which must be consider before the ideas and solutions generated. This boundary condition marks the end of the research stage.

Once this boundary condition is considered, the Development stage begins, with the process continuing in a similar fashion to the Chesbrough model, with a number of ideas and concepts moving both inwards and outwards of the model during the process. Ideas are born, and tested and either progress, move into other applications or are removed. The ideas that move out of the funnel are similar to "Technology Spin-offs" but in this case are particular ideas for use in a specific external application (ex. Hand washing guide for developing regions). When a number of solutions are developed, they undergo a refinement process, in which they are validated, optimised and prepared for public dissemination.

This is followed by the dissemination of the developed ideas both internally in the established project ecosystem and 
external to the world at large. Internal ecosystem dissemination includes distribution to other teams, groups or organisations attempting to tackle the same issue or larger deployment partners such as the WHO in the case of open source ventilators. Dissemination to non-ecosystem partners then occurs when the idea is in effect release for implementation by the world at large. While this dissemination is generally non targeted, there may be dedicated dissemination channels to allow the direct transmission of ideas and solutions to locations groups that are in acuate need of the solutions (Such as refugee camps for the ventilator systems). Visualising this process gives a form of inverted Open Innovation process funnel, mapping the process visually. This visual representation can be seen in Figure $7^{[46]}$, with the working title of the "Inverted Open Innovation Distribution Model".

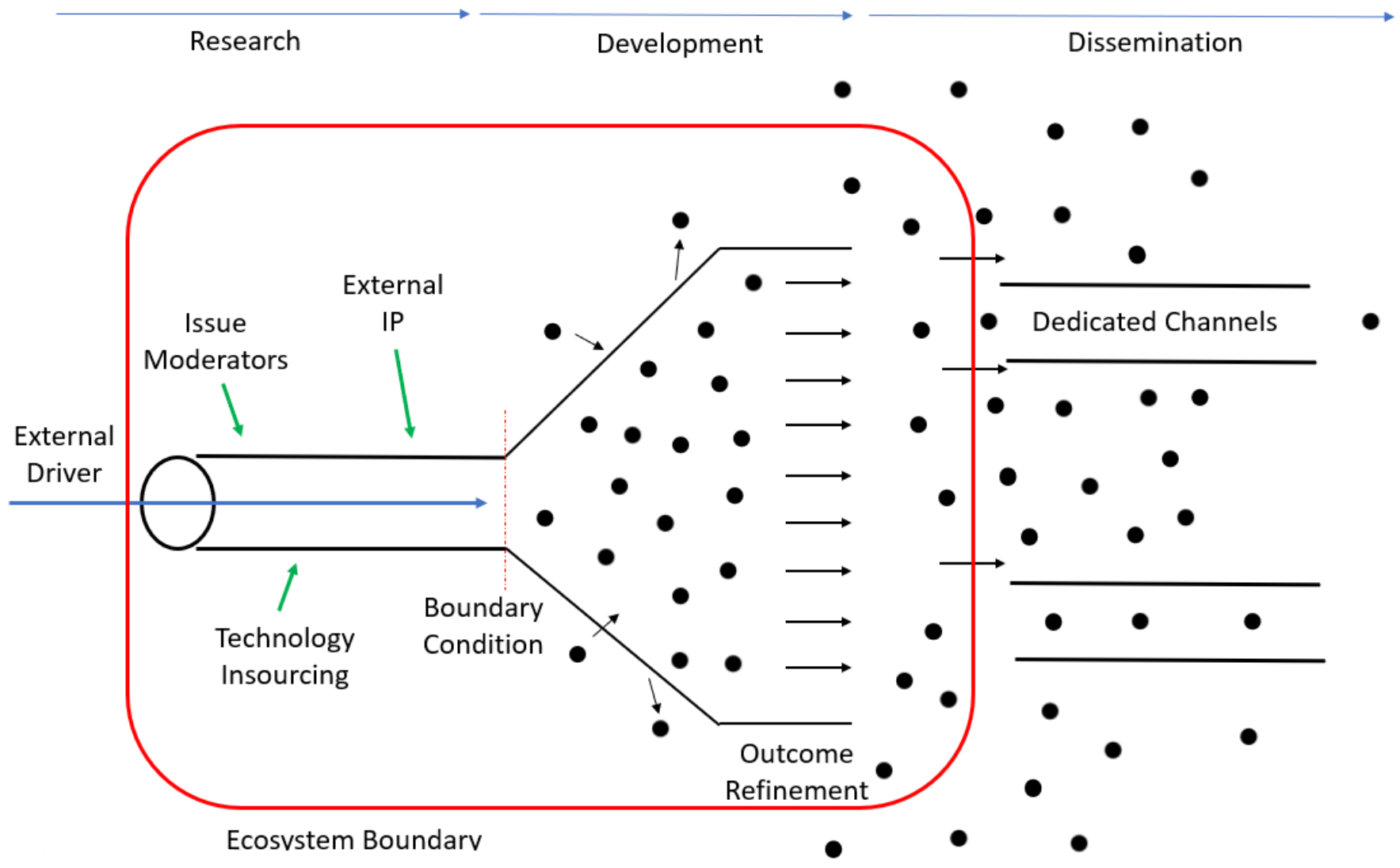

Figure 7. The Inverted Open Innovation Distribution Model Mapping the TeamOSV Process [46]

\section{Conclusion}

It is clear that the process of developing a highly regulated medical device remotely with a diverse non expert team is a complex task in what is a highly regulated field, as such is not to be taken lightly, but there does exist significant potential for deploying similar teams, approaches and structures to help tackle global issues in the future. These new forms of open innovation led open source projects can react with speed and innovative capacity on par with any large corporation or national government. TeamOSV activities clearly showed that committed groups of volunteers can effect significant 
change and impact through the utilisation of innovative approaches online, even in fields in which this approach is new. There does however exist some areas of particular importance to help increase the chances of success, namely the development of good organisational structure early it the project, mechanisms to help deal with significant external pressures on the process and excellent understanding and management of the human aspect of the project. Further work on assessing the internal interactions of volunteers, the impact of significant external factors on project pace and development of an organisational framework for similar projects is required. The first attempt at mapping the process resulted in the proposed "Inverted Open Innovation Distribution Model", which require further development and refinement, along with validation on similar projects. Traditional organisations and process must be included in the development of these interventions going forward, to achieve the potential of this approach. Approach specific policies, regulations, mechanisms and pathways will help increase the potential impact of not only other open source ventilator project, but other emergency response projects in the future.

\section{References}

1. a, b, c, d, e (2020). TeamOSV Homepage.

2. `Johns Hopkins University of Medicine. (2020). Coronavirus Resource Centre [Internet].

3. ^World Health Organization. (2020). World Health Organization: Critical preparedness, readiness and response actions for COVID-19: interim guidance.

4. ^Ramsey L. (2020). ospitals could be overwhelmed with patients and run out of beds and ventilators as the coronavirus pushes the US healthcare system to its limits.. Business Insider.

5. ^. (2020). COVID-19: too little, too late?. The Lancet, vol. 395 (10226), 755. doi:10.1016/s0140-6736(20)30522-5.

6. ' Gabriele Ciminelli, Sílvia Garcia-Mandicó. (2020). COVID-19 in Italy: An Analysis of Death Registry Data. doi:10.1093/pubmed/fdaa165.

7. ^Xinzhi Zhang, Martin I. Meltzer, Pascale M. Wortley. (2006). FluSurge-A Tool to Estimate Demand for Hospital Services during the Next Pandemic Influenza. Med Decis Making, vol. 26 (6), 617-623. doi:10.1177/0272989x06295359.

8. ${ }^{a}, b, c, d, e, f(2020)$. Open Source Ventilator Ireland.

9. a, b, c, d(2020). OpenLung Gitlab.

10. ^(2020). Irish engineers and doctors join forces in the face of Covid-19.Silicon Republic.

11. `Shane Keaveney, Colin Keogh, Luis Gutierrez-Heredia, Emmanuel G. Reynaud. (2016).Applications for advanced 3D imaging, modelling, and printing techniques for the biological sciences. doi:10.1109/vsmm.2016.7863157.

12. 'Aviva Cohen, Lizbeth Goodman, Shane Keaveney, Colin Keogh, et al. (2017). Sustaining a caring relationship at a distance: Can haptics and 3D technologies overcome the deficits in 2D direct synchronous video based communication?. doi:10.1109/vsmm.2017.8346290.

13. ${ }^{a}, \mathrm{~b}$ Luis Gutierrez-Heredia, Colin Keogh, Emmanuel G. Reynaud. (2018).Assessing the Capabilities of Additive Manufacturing Technologies for Coral Studies, Education, and Monitoring. Front. Mar. Sci., vol. 5. 
doi:10.3389/fmars.2018.00278.

14. `Colin Keogh, Vincent Hyland, Rick Nassar. (2017).3D rocks - Augmented reality Islands for Skellig Kerry region. doi:10.1109/vsmm.2017.8346267.

15. `Andre Laplume, Gerald C. Anzalone, Joshua M. Pearce. (2015). Open-source, self-replicating 3-D printer factory for small-business manufacturing. Int J Adv Manuf Technol, vol. 85 (1-4), 633-642. doi:10.1007/s00170-015-7970-9.

16. ^Joshua M. Pearce. (2020). A review of open source ventilators for COVID-19 and future pandemics. F1000Res, vol. 9 , 218. doi:10.12688/f1000research.22942.1.

17. ^Emily Petersen, Joshua Pearce. (2017). Emergence of Home Manufacturing in the Developed World: Return on Investment for Open-Source 3-D Printers. Technologies, vol. 5 (1), 7. doi:10.3390/technologies5010007.

18. `Tulsi R. Damase, Daniel Stephens, Adam Spencer, Peter B. Allen. (2015).Open source and DIY hardware for DNA nanotechnology labs. J Biol Methods, vol. 2 (3), 24. doi:10.14440/jbm.2015.72.

19. 'Corinne Dally, Daniel Johnson, Moriah Canon, Sarah Ritter, et al. (2015).Characteristics of a 3D-printed prosthetic hand for use in developing countries. doi:10.1109/ghtc.2015.7343956.

20. `Shane Oberloier, Joshua Pearce. (2017). General Design Procedure for Free and Open-Source Hardware for Scientific Equipment. Designs, vol. 2 (1), 2. doi:10.3390/designs2010002.

21. 'Henry Chesbrough. (2020). To recover faster from Covid-19, open up: Managerial implications from an open innovation perspective. Industrial Marketing Management, vol. 88 , 410-413. doi:10.1016/j.indmarman.2020.04.010.

22. ^(202 0). Open Source Medical Supplies Group.

23. ^(2020). OSV Slack Channel.

24. ^OSVX Slack Channel.

25. ^(2020). DIY Beatmungsgeraet.

26. ^(2020). Inspire OpenLung.

27. ^(2020). Velocity Research.

28. 'Arjen M. Dondorp, Muhammad Hayat, Diptesh Aryal, Abi Beane, et al. (2020).Respiratory Support in COVID-19 Patients, with a Focus on Resource-Limited Settings. doi:10.4269/ajtmh.20-0283.

29. ^DANIELLE KIRSH. (2020). Ford partners with GE Healthcare to make 50k ventilators in 100 days.Mass Device.

30. a, b(2020). TeamOSV Guidance.

31. ^(2020). TeamOSV BCT.

32. ^(2020). Coronavirus: Schools, colleges and childcare facilities in Ireland to shut. The Irish Times.

33. ${ }^{a, b}$ (2020). Gov.ie - Public Health Measures in place until 12 April to prevent spreading COVID-19.

34. a, b(2020). "There Aren't Enough Ventilators to Cope With the Coronavirus". New York Times.

35. ^UK Government. (2020). "Health Secretary Matt Hancock's Sunday Telegraph Op-Ed “.

36. 'UK Government. (2020). "Specification for ventilators to be used in UK hospitals during the coronavirus (COVID-19) outbreak".

37. ^(2020). Muddled thinking punctures plan for British ventilator ". Financial Times.

38. " (2020). "70 years of innovation: how F1 came together for Project Pitlane".

39. ^Oxford University. (2020). OxVent. 
40. ^Redbull F1. (2020). BlueSky Ventilator.

41. 'Reuters. (2020). "UK cancels order for simple ventilators, needs more complex ones: source”. Reuters.

42. ${ }^{a, b, c}$ Robert L. Read, Keeshan Patel, Juan E. Villacres Perez and Avinash Baskaran. (2020).Analysis of Open Source COVID-19 Pandemic Ventilator Projects.

43. a, b, c Henry Chesbrough. (2020). To recover faster from Covid-19, open up: Managerial implications from an open innovation perspective. Industrial Marketing Management, vol. 88 , 410-413. doi:10.1016/j.indmarman.2020.04.010.

44. 'Henry Chesbrough. (2012). Open Innovation: Where We've Been and Where We're Going.Research-Technology Management, vol. 55 (4), 20-27. doi:10.5437/08956308x5504085.

45. ${ }^{a}, \mathrm{~b} H e n r y$ Chesbrough, Adrienne Kardon Crowther. (2006). Beyond high tech: early adopters of open innovation in other industries. R\&D Management, vol. 36 (3), 229-236. doi:10.1111/j.1467-9310.2006.00428.x.

46. ${ }^{a, b, ~ c}$ Colin Keogh. (2021). Inverted Open Innovation Distribution Model. Qeios. doi:10.32388/3M2W5R. 